\title{
Transition from binary processes to multifragmentation in quantum molecular dynamics for intermediate energy heavy ion collisions
}

\author{
Li Zhuxia, ${ }^{*}$ C. Hartnack, H. Stöcker, and W. Greiner \\ Institut für Theoretische Physik der J. W. Goethe, Universität Frankfurt, Frankfurt, Germany
}

(Received 19 July 1990)

\begin{abstract}
We study the transition from fusion-fission phenomena at about $20 \mathrm{MeV} /$ nucleon multifragmentation at $100-200 \mathrm{MeV} /$ nucleon in the reaction ${ }^{16} \mathrm{O}+{ }^{80} \mathrm{Br}$ employing the quantum molecular dynamics model. The time evolution of the density and mass distribution, the charged-particle multiplicity, and spectra as well as angular distributions of light particles are investigated. The results exhibit the transition of the disassembly mechanism, but no sharp change is found. The results are in good agreement with recently measured $4 \pi$ data.
\end{abstract}

\section{INTRODUCTION}

Many experimental and theoretical efforts have been made to study the disassembly of hot and dense systems produced in intermediate energy heavy ion collisions. It turns out that the situation is very complicated. Many problems are still open. One of the most interesting problems is the onset of multifragmentation [1]. Recently, the transition from binary decay to multifragmentation has been observed in high-multiplicity heavy ion induced reactions between 20 and $200 \mathrm{MeV} /$ nucleon [2]. This led us to the present work.

In order to describe the formation of clusters in heavy ion reactions, a dynamical model must include manybody correlations and fluctuations.

In a previous work we presented an extension of the quantum molecular dynamics (QMD) model [3], where a Pauli potential $[4,5]$ is used to simulate the Pauli exclusion principle. This initialization procedure [6] is supplemented by a cooling mechanism that removes artificial excitation energy. In this way the QMD model that successfully describes heavy ion collisions in the few hundred $\mathrm{MeV} /$ nucleon regime may be applied to lowerenergy reactions down to about $10 \mathrm{MeV} /$ nucleon [3].

Since the QMD model incorporates classical $N$-body correlations and fluctuations, it is able to describe cluster formation on a semiclassical level.

In this publication we use the QMD model to study the transition from binary decay to multifragmentation. Especially we will investigate the question of which observables distinguish between those two decay modes. It will be shown here that apart from the charged-particle multiplicity and the mass distribution, other quantities, e.g., nucleon spectra, angular distribution of protons, and complex light fragments, may help in the study of the transition to the multifragmentation decay mode.

We have decided to present the theoretical analysis for the system ${ }^{16} \mathrm{O}+{ }^{80} \mathrm{Br}$ in order to allow for a direct comparison with the stimulating experimental results of Ref. [2]. Konopka, Peilert, Stöcker, Greiner, and Neise [7] and Peilert, Randrup, Stöcker, and Greiner [5] have indeed shown that the transition is more sharply localized in energy for symmetric systems.

In Sec. II we will recall our model briefly. In the third part some results will be shown, and finally we discuss some problems concerning the model.

\section{OUTLINE OF THE MODEL}

The trajectories of $N$ particles represented by Gaussian wave packets are described by a set of canonical equations of motion

$$
\dot{\mathbf{r}}_{i}=\frac{\partial H}{\partial \mathbf{p}_{i}}, \quad \dot{\mathbf{p}}_{i}=-\frac{\partial H}{\partial \mathbf{r}_{i}}, \quad i=1, \ldots, N .
$$

The Hamiltonian used in (1) is given by

$$
\begin{gathered}
H=\sum_{i} \frac{\mathbf{p}_{i}^{2}}{2 m}+\frac{1}{2} \sum_{i \neq j}\left[V_{Y}(i, j)+V_{P}(i, j)+V_{C}(i, j)\right. \\
\left.+V_{\mathrm{dd}}(i, j)+V_{\mathrm{md}}(i, j)\right]
\end{gathered}
$$

with $V_{Y}(i, j)$ the Yukawa interaction, $V_{P}(i, j)$ the Pauli potential, $V_{C}(i, j)$ the Coulomb interaction, $V_{\mathrm{md}}(i, j)$ the momentum-dependent force, and $V_{\mathrm{dd}}(i, j)$ the densitydependent (Skyrme) interaction. The force parameters are the same as given in [3].

A cooling mechanism is introduced into the initialization phase. After the positions and momenta of the nucleons in phase space are generated randomly, the nuclei are propagated according to damped equations of motion [3]. This removes most of the artificial excitation energy generated by the above procedure and leads to "cold" computational nuclei.

The minimum-space span method [8] is used to determine the clusters in the exit channel. In this algorithm nucleon pairs separated by less than $3 \mathrm{fm}$ are considered to belong to the same cluster. At every time step we determine the (preliminary) cluster distribution. Thus the time evolutions of mass, charge, position, and momentum of each fragment or single nucleon are obtained. 


\section{RESULTS}

\section{A. The time evolution of density}

In order to have some general concept of the disassembly of nuclei in nuclear reactions, we first study the time evolution of the mean density [9], defined as

$$
\langle\rho\rangle=\frac{\int d \mathbf{r} \rho^{2}}{\int d \mathbf{r} \rho}
$$

and the strongly connected number of particles remaining in the system.

Figure 1 shows the evolution of $\langle\rho\rangle$ at different beam energies between 25 and $200 \mathrm{MeV} /$ nucleon. The highest density is reached after $10-30 \mathrm{fm} / c$ for all beam energies depending on projectile velocity. At $t=50 \mathrm{fm} / c$ all curves reach a first minimum, but further develop in rather different ways.

At $200 \mathrm{MeV} /$ nucleon the density drops to less than half of the initial value, whereas in the $25-\mathrm{MeV} /$ nucleon case the system (incompletely) fuses and then undergoes vibrations predominantly in monopole mode. Thus, there is a big difference in the density evolution in medium- (100-200 MeV/nucleon) and low-energy (25-50 MeV/nucleon) reactions. In the first case the density drops quickly at $t=20-50 \mathrm{fm} / c$ due to the very fast expansion of the system. In this stage light fragments are emitted $(\approx 33$ nucleons for $200 \mathrm{MeV} /$ nucleon and $\approx 17$ nucleons for $100 \mathrm{MeV} /$ nucleon). On the contrary, for $25 \mathrm{MeV} /$ nucleon the system fuses and only small quantities of light particles are emitted.

Strongly connected to the density evolution is the number of particles remaining in the vicinity of the center of mass. As a measure we count the number of nucleons contained in a sphere of radius $9.25 \mathrm{fm}$ about the c.m. as a function of time. This is displayed in Fig. 2 for central collisions at four different energies. Initially, all $96 \mathrm{nu}-$ cleons are inside the sphere. Again, if we look at the particle number after $170 \mathrm{fm} / c$, the behavior discovered for

\section{time evolution of density}

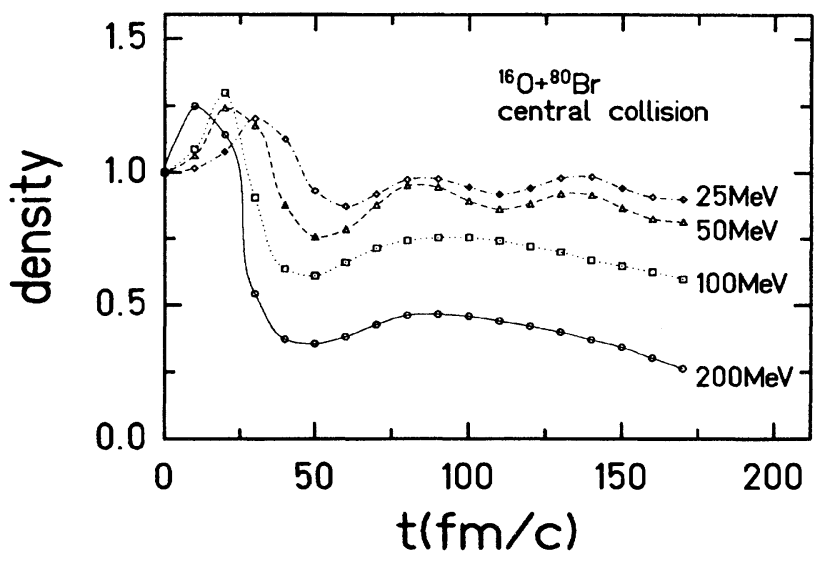

FIG. 1. The time evolution of mean density at beam energy $25,50,100$, and $200 \mathrm{MeV} /$ nucleon, respectively.

\section{particles remain}

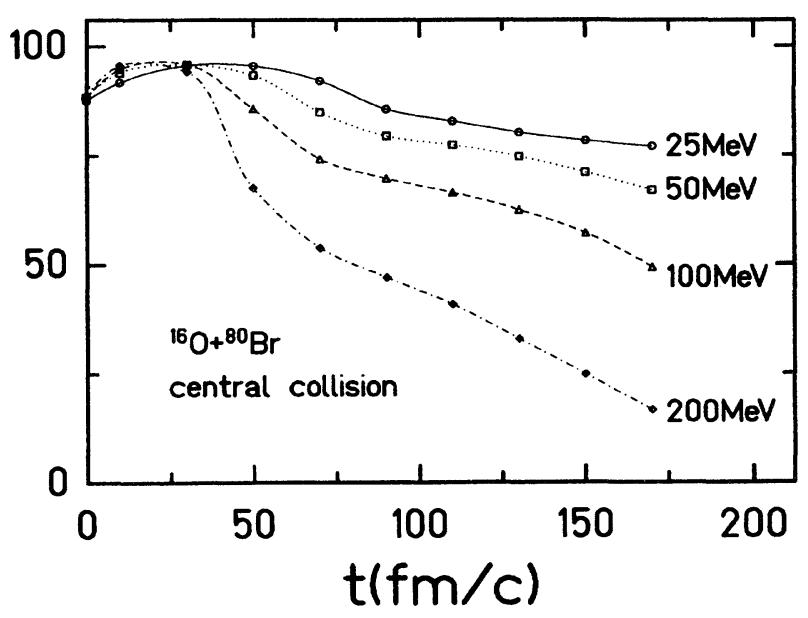

FIG. 2. The time evolution of the number of particles remaining in the volume with radius $9.25 \mathrm{fm}$ centered at the center of mass for a central collision. The incident energies are $25,50,100$, and $200 \mathrm{MeV} /$ nucleon.

the mean density shows up. Whereas for 200 $\mathrm{MeV} /$ nucleon only $10 \%$ of the particles are at the center of mass, nearly $80 \%$ of particles remain in the sphere for $25 \mathrm{MeV} /$ nucleon.

\section{B. The time evolution of the mass distribution}

In the previous section we investigated the general behavior of the temporal evolution of the heavy ion collision. To get more detailed information, we now look at the temporal evolution of the mass distribution in the reaction oxygen on bromine at $200 \mathrm{MeV} /$ nucleon, shown in Fig. 3.

At $30 \mathrm{fm} / c$ the nuclei merge into one big cluster and several nucleons and light fragments are emitted. After $60 \mathrm{fm} / c$ approximately 30 nucleons and several complex clusters have already separated and left one big cluster with nearly half the target mass. A valley in the region of one-fourth of target mass shows up. This valley, howev$\mathrm{er}$, is filled between 90 and $120 \mathrm{fm} / c$ due to the slow breakup of the residues. Big clusters $\left(A \approx A_{T} / 2\right)$ now have only negligible yield. Actually, after $200 \mathrm{fm} / c$ there is no cluster with mass larger than half of the target mass.

This should be compared to the evolution at 25 $\mathrm{MeV} /$ nucleon displayed in Fig. 4. Here, after $40 \mathrm{fm} / \mathrm{c}$ the nuclei have fused. Only few nucleons are emitted in this stage. From then on several nucleons are emitted from the compound system followed by the emission of some very light clusters. After about $100 \mathrm{fm} / c$ the system seemingly has two decay modes: first, fission into two pieces, one of intermediate size and the other well below the target mass. Second, evaporation of several fragments. This leads to the double-peak behavior on the heavy-mass side of the mass distribution. At the end of the reaction, after $400 \mathrm{fm} / c$, the shape of the mass distri- 

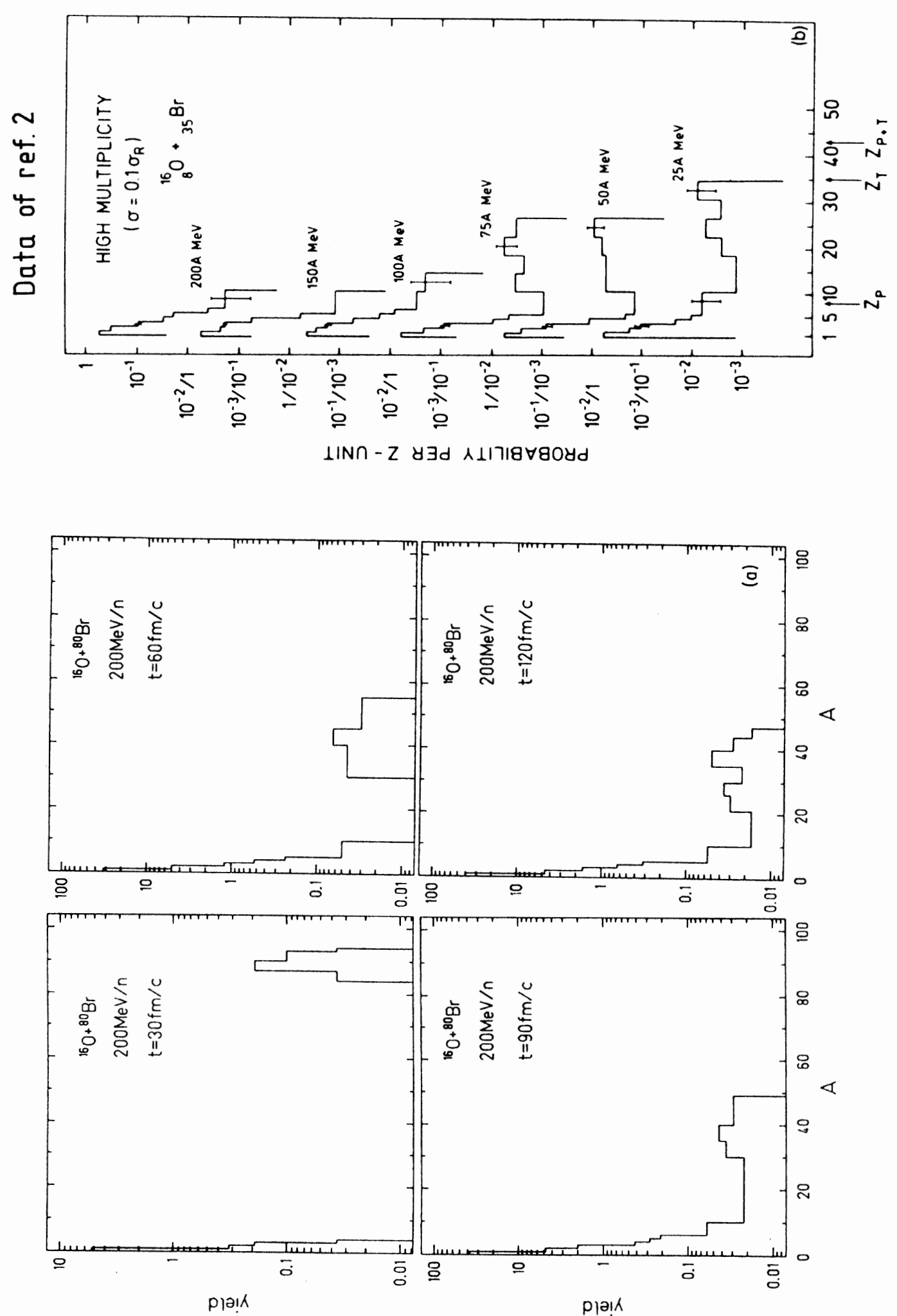

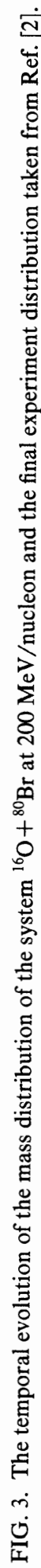



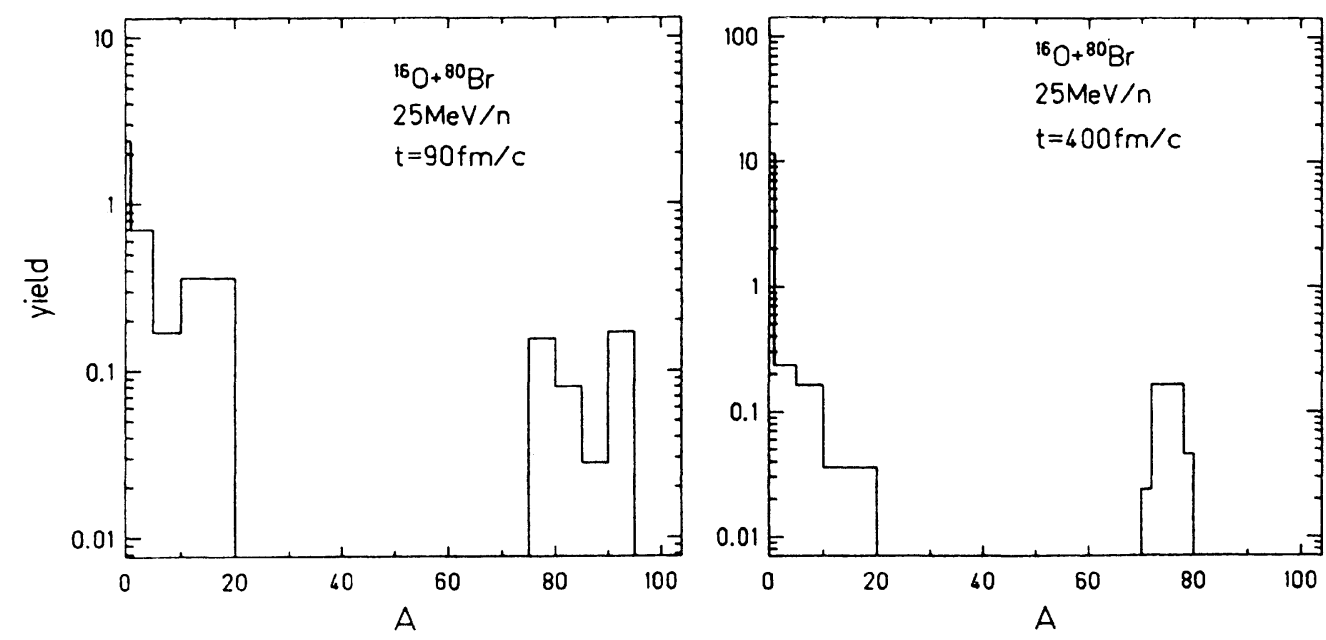

FIG. 4. Same as Fig. 3, but for incident energy $25 \mathrm{MeV} /$ nucleon. The mass distribution at $30 \mathrm{fm} / \mathrm{c}$ is not shown, since it only consists of a single line at mass 96 .

bution has completely changed due to further evaporation of light particles and sequential breakup of intermediate-mass fragments.

The source of the intermediate-mass fragments in intermediate energy heavy ion collisions has attracted much interest, from both the experimental and theoretical side [10]. From our study of the temporal evolution of the mass distribution we deduce that at low energies intermediate-mass fragments are predominantly created by sequential binary decay, whereas at higher energies those clusters stem from multifragmentation-like processes. To make a further check of the validity of this conclusion, we calculated the total yield of intermediate mass fragments (IMF), which we define as clusters in the mass range $5 \leq A \leq 40$. The onset of multifragmentation is to be expected in the beam energy range considered here. The mean multiplicity of IMF for different energies are given in Table I (for central collisions). The decrease of the $M_{\text {IMF }}$ at beam energy 50-100 MeV/nucleon comparing to $25 \mathrm{MeV} /$ nucleon is due to the increase of the high-multiplicity events, where many very light particles $(p, n, d, t, \ldots)$ are emitted leaving one heavy cluster with mass $\approx 70$ at $50 \mathrm{MeV} /$ nucleon and $\approx 60$ at 100 $\mathrm{MeV} /$ nucleon. These events have no contribution to the IMF yield, but contribute to the light complex fragments.

TABLE I. Multiplicity of intermediate-mass fragments (IMF) for central collisions at different energies.

\begin{tabular}{cc}
\hline \hline & $M_{\text {IMF }}$ \\
Multiplicity & $\begin{array}{c}\text { Beam energy } \\
(\mathrm{MeV} / \text { nucleon })\end{array}$ \\
$\approx 0.9$ & 25 \\
$\approx 0.7$ & $50-100$ \\
$\approx 1.5$ & 200 \\
\hline \hline
\end{tabular}

\section{Energy and impact-parameter dependence of charged-particle multiplicity}

Figure 5 displays the total charged-particle multiplicity in central collisions of ${ }^{16} \mathrm{O}$ on ${ }^{80} \mathrm{Br}$ at beam energies ranging from 25 to $200 \mathrm{MeV} /$ nucleon. It has to be considered as a two-dimensional scatter plot of the number of events versus beam energy and multiplicity of charged particles. Thus the numbers plotted in the figure are the number of events with a given multiplicity at a given energy. The curve shows the profile of the highest charged-particle multiplicity at different beam energies. The general behavior is very similar to the results of an emulsion experiment by Jakobsson et al. [2]. There seems to exist a kink at about $70 \mathrm{MeV} /$ nucleon at the profile curve. This corresponds to the change of the time evolution of the mean density in Fig. 1 and probably reflects a change in the breakup mechanism at this energy. In Fig. 6 we show the impact-parameter dependence of the charged-particle multiplicity in the beam energy range from 25 to 200 $\mathrm{MeV} /$ nucleon. It seems that the impact parameter dependence of the average charged-particle multiplicity is much stronger at higher energy than at lower energy. At beam energies as low as $25 \mathrm{MeV} /$ nucleon the multiplicity is not sensitive to impact parameter anymore as long as $b$ is not too large. In view of this it seems very questionable to use charged-particle multiplicity as a meter for the impact parameter at low energies.

However, in our model protons and neutrons are only distinguished by the Coulomb interaction. The symmetry energy is completely neglected in the Hamiltonian (3). Introducing the symmetry energy may have some influence on the results, but surely it will not change their general character.

\section{Spectra and angular distributions of light fragments}

Figure 7 displays the laboratory proton spectra of the reaction ${ }^{16} \mathrm{O}+{ }^{80} \mathrm{Br}$ at incident energies of 25,50 , and 200 


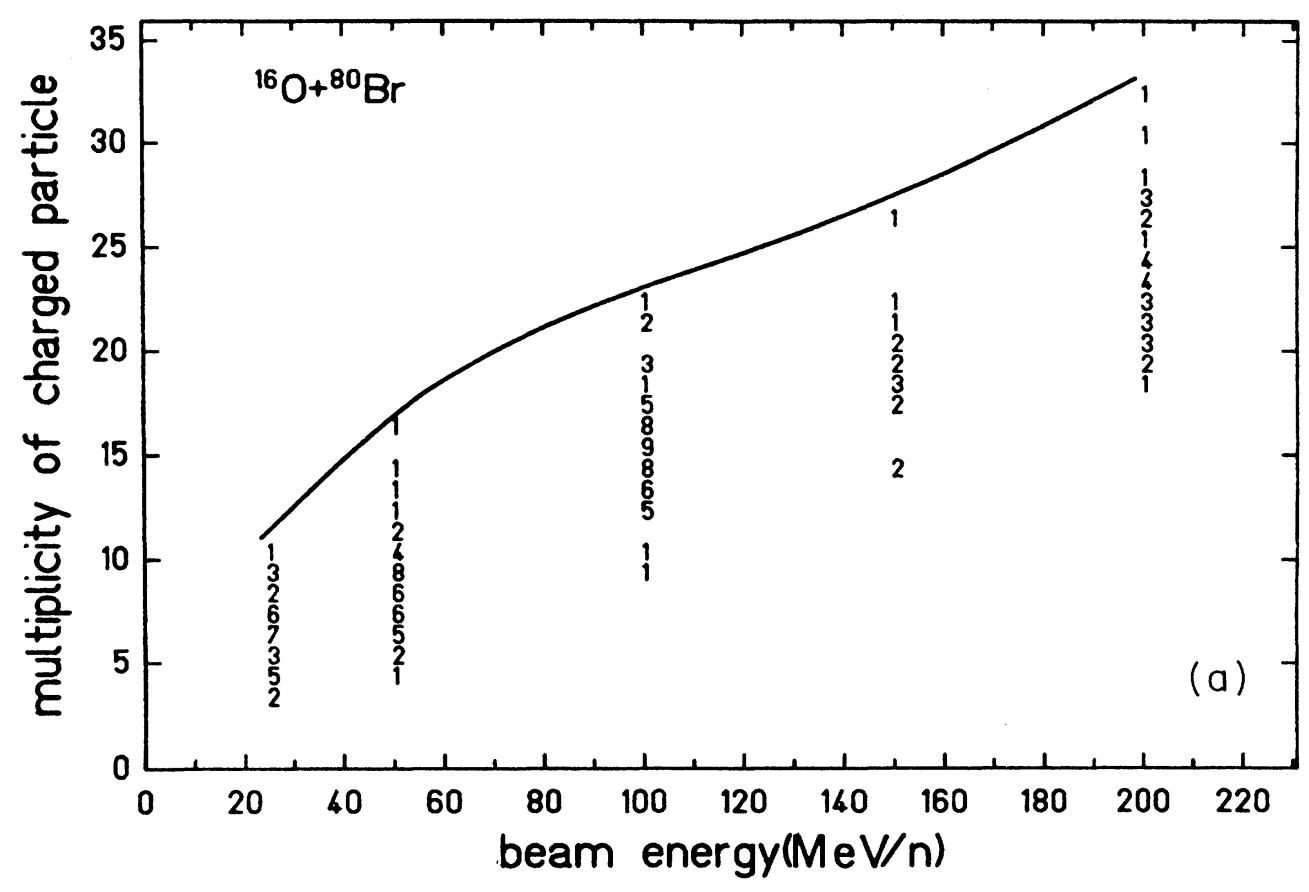

Data of ref. 2

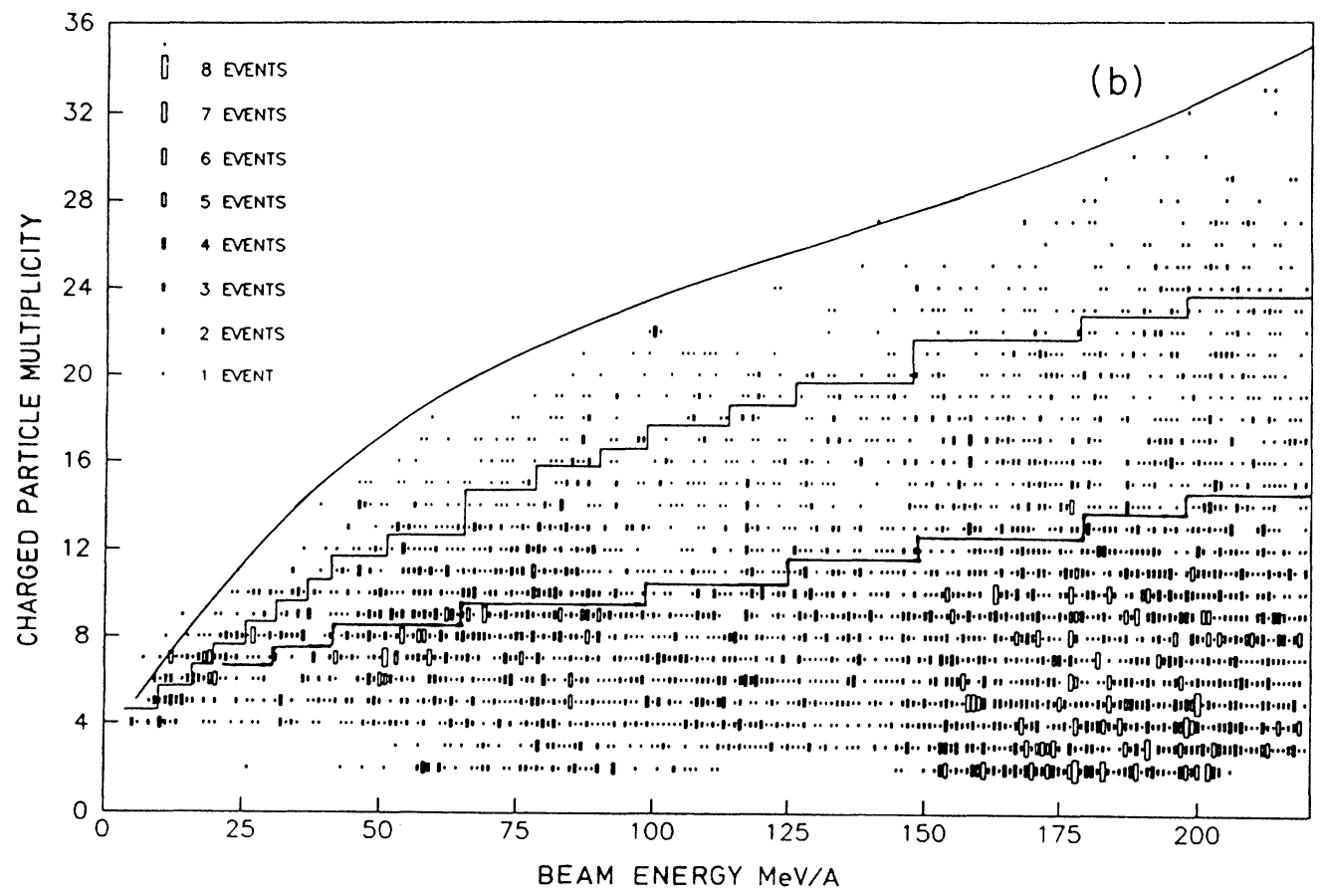

FIG. 5. Scatter plot of the number of events versus beam energy and total charged-particle multiplicity and experimental results taken from Ref. [2]. 


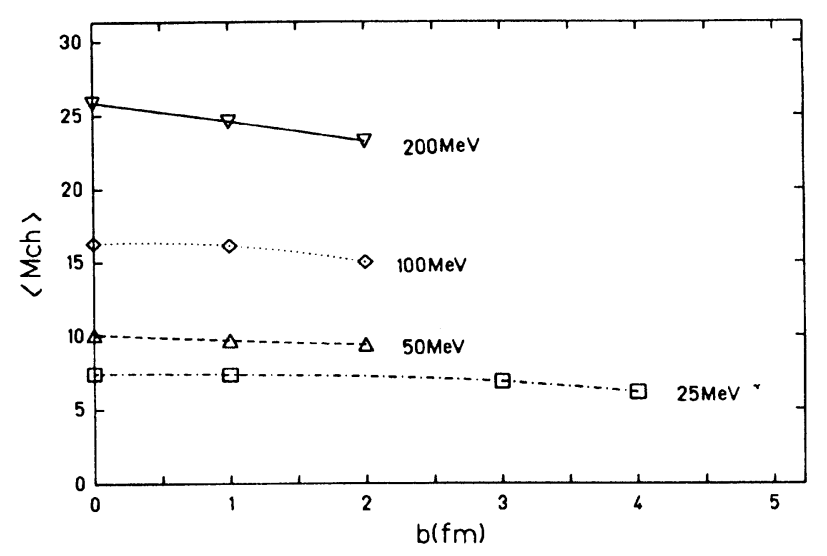

FIG. 6. Impact-parameter dependence of the average charged-particle multiplicity.

$\mathrm{MeV} /$ nucleon. The three spectra do not differ very much for low proton energies $(\leq 30 \mathrm{MeV})$. From this we deduce that those protons are evaporated from effective sources of almost same temperature, no matter how high the incident energy is. In order to get more information about the proton source, we studied the angular distribution of the protons for different energy cuts. In Fig. 8 we show the double-differential cross section in the c.m. system for different beam energies. The low-energy protons predominantly stem from evaporation processes. Let us concentrate first on the $25-\mathrm{MeV} /$ nucleon collision. Here, the angular distribution of the low-energy protons $\left(0<E_{P} \leq 100 \mathrm{MeV}\right)$ shows two components. This implies that these protons are evaporated by two distinct sources moving in almost opposite direction. With increasing beam energy, however, this angular distribution becomes less structured until at $200 \mathrm{MeV} /$ nucleon the protons appear to stem from one single source only. This can be understood as follows: The protons are emitted by many sources moving in different directions, and consequently the structures in the angular distribution are smeared out. Therefore, the protons seem to be emitted from one source centered at the center of mass.

The angular distributions for higher-energy protons are peaked forward for low incident energy and sideward for high incident energy. These fast protons mainly stem from the early stage of the reaction. They carry most of the initial momentum and kinetic energy away.

The angular distribution of high-energy protons $\left(E_{P}>100 \mathrm{MeV}\right)$ in the $200-\mathrm{MeV} /$ nucleon reaction show the sideward flow effect. This is consistent with early experiments [11].

The impact parameter dependence of the proton spectra calculated with QMD are consistent with kinematical considerations: In the lower-energy part of the spectrum $\left(E_{P} \leq E_{\text {beam }} / 2\right)$ there is an enhancement of the proton yield for small impact parameters compared to more peripheral collisions. The spectra and yields increase with increasing beam energy.

The differential cross section $d \sigma / d \cos \theta$ shown in Fig. 9 displays the enhancement at middle angles for high

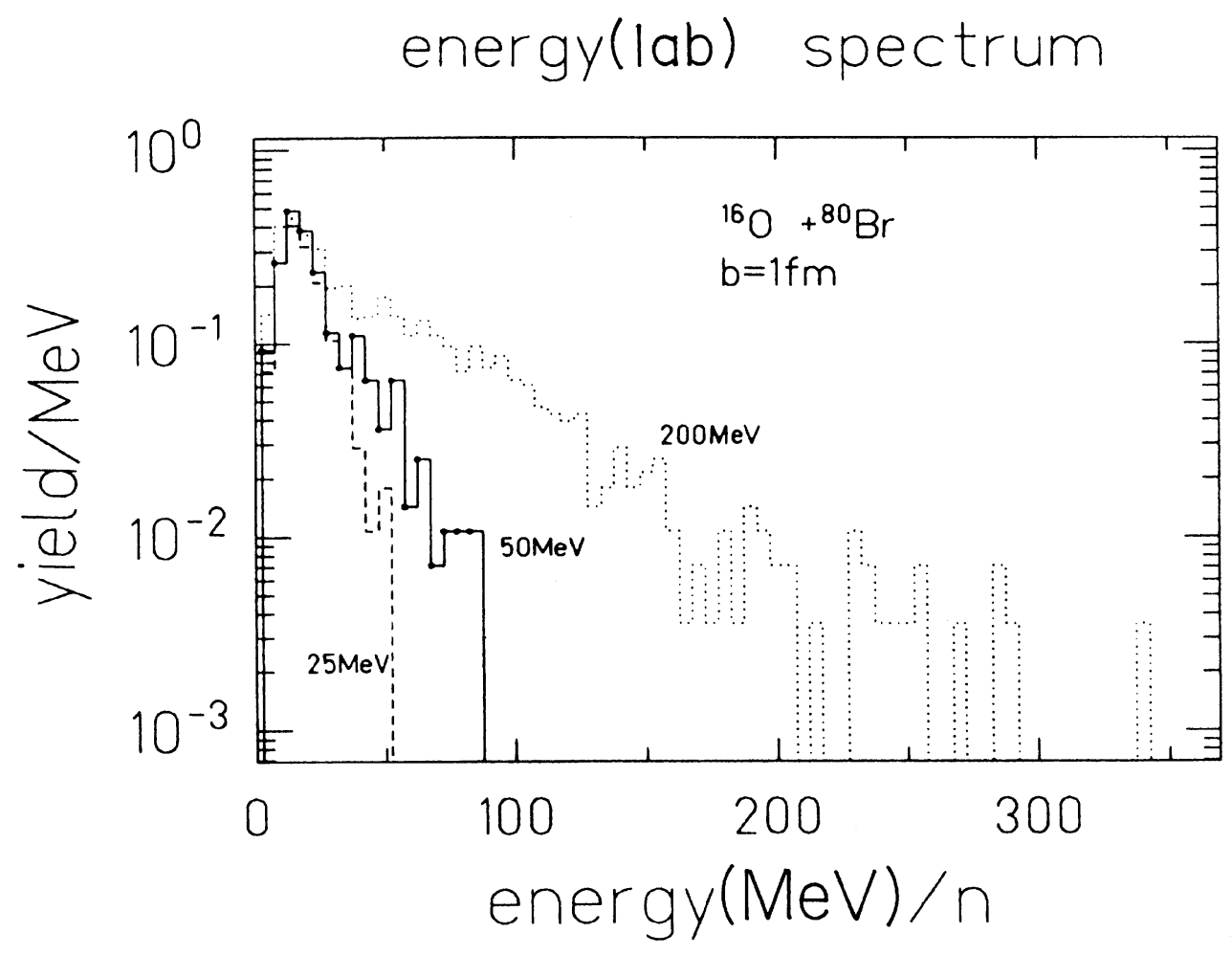

FIG. 7. Proton spectra in the laboratory system for central collisions of ${ }^{16} \mathrm{O}+{ }^{80} \mathrm{Br}$ at $25,50,100$, and $200 \mathrm{MeV} / \mathrm{nucleon}$. 
beam energy. The distribution for $200 \mathrm{MeV} /$ nucleon shows similar character as early measurements of the reactions ${ }^{12} \mathrm{C}+\mathrm{Ag}$ and $\mathrm{Cl}[1]$. In contrary, for lower beam energies the differential cross section is forward peaked.

In Fig. 10 we compare the spectra of protons and light complex fragments (LCF) at $200 \mathrm{MeV} /$ nucleon. For low particle energies both spectra look very similar. For higher energies, however, light particle emission is strongly suppressed compared to protons. In fact, the ICF spectrum is linked to about three quarters of the beam energy. This implies that some nucleons at the Fermi surface are emitted at the very beginning of the reaction, carrying a large fraction of the available kinetic energy away. The light complex fragments are emitted later on. The double-differential cross sections of light complex fragments at beam energy $200 \mathrm{MeV} /$ nucleon given in Fig. 11 show even more pronounced side flow at middle angles for fast light fragments compared with protons.

Now let us summarize this part. The spectra and angular distributions of protons show some remarkable characteristics in the beam energy range between 25 and $200 \mathrm{MeV} /$ nucleon. Changes in the character of spectra and angular distribution reflect a change in the reaction mechanism from binary processes to multifragmentation.

These observables provide us with a valuable tool to investigate the transition to multifragmentation and strongly support the analysis of multiplicities.

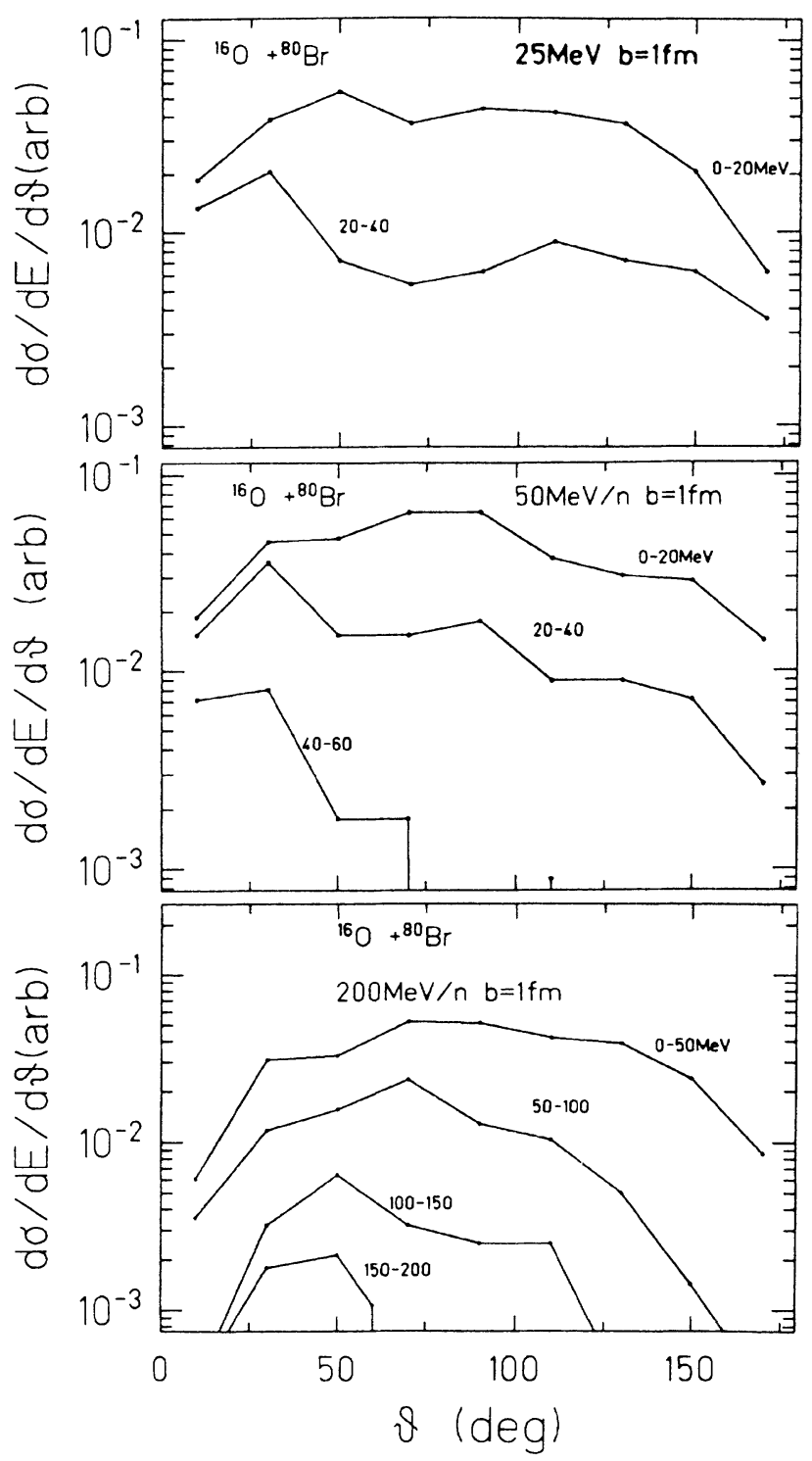

FIG. 8. The double-differential cross section in the c.m. system for beam energies 25,50 , and $200 \mathrm{MeV} /$ nucleon.

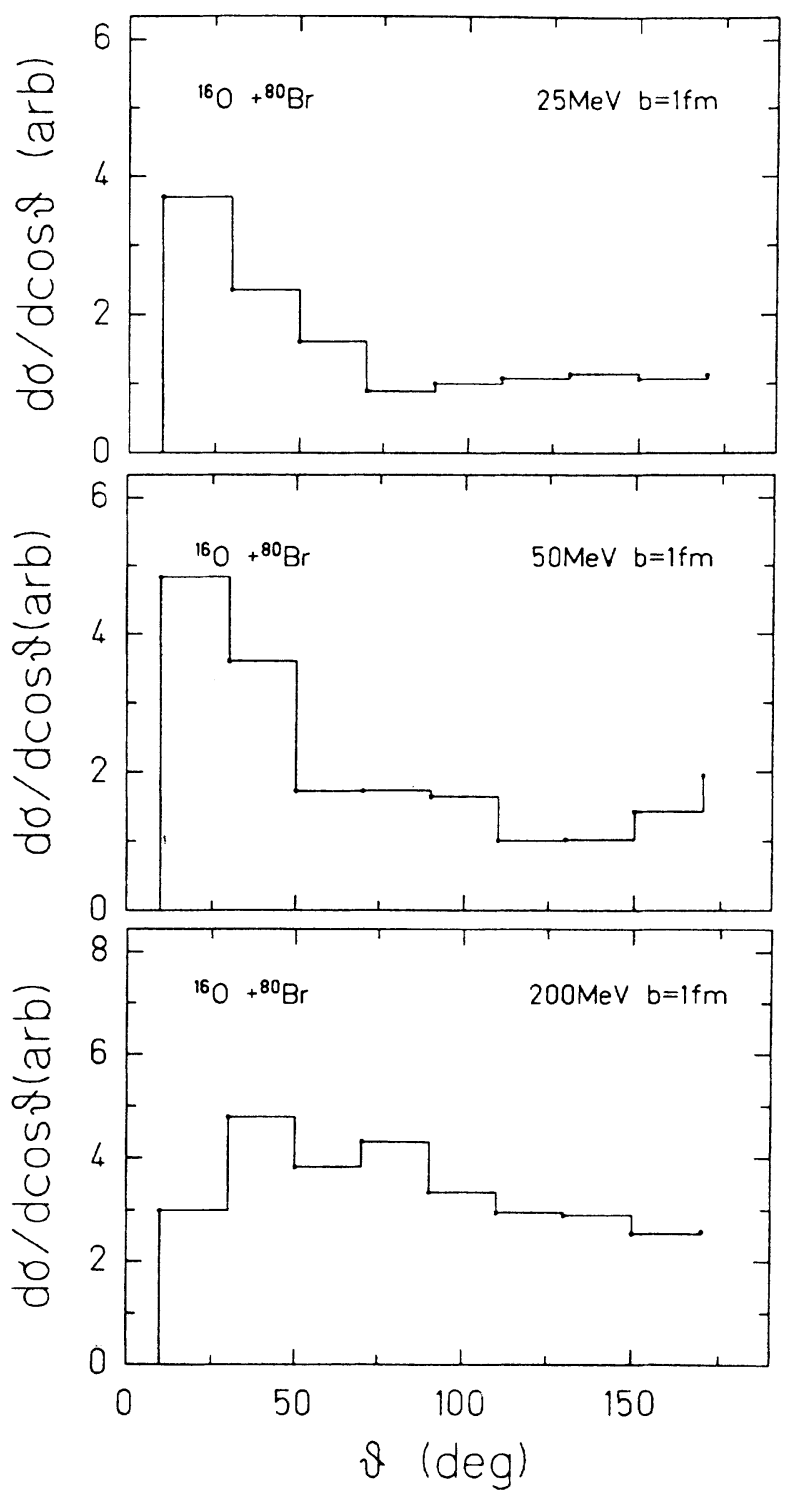

FIG. 9. The proton differential cross section $d \sigma / d \cos \theta$ for beam energies of 25,50 , and $200 \mathrm{MeV} /$ nucleon. 


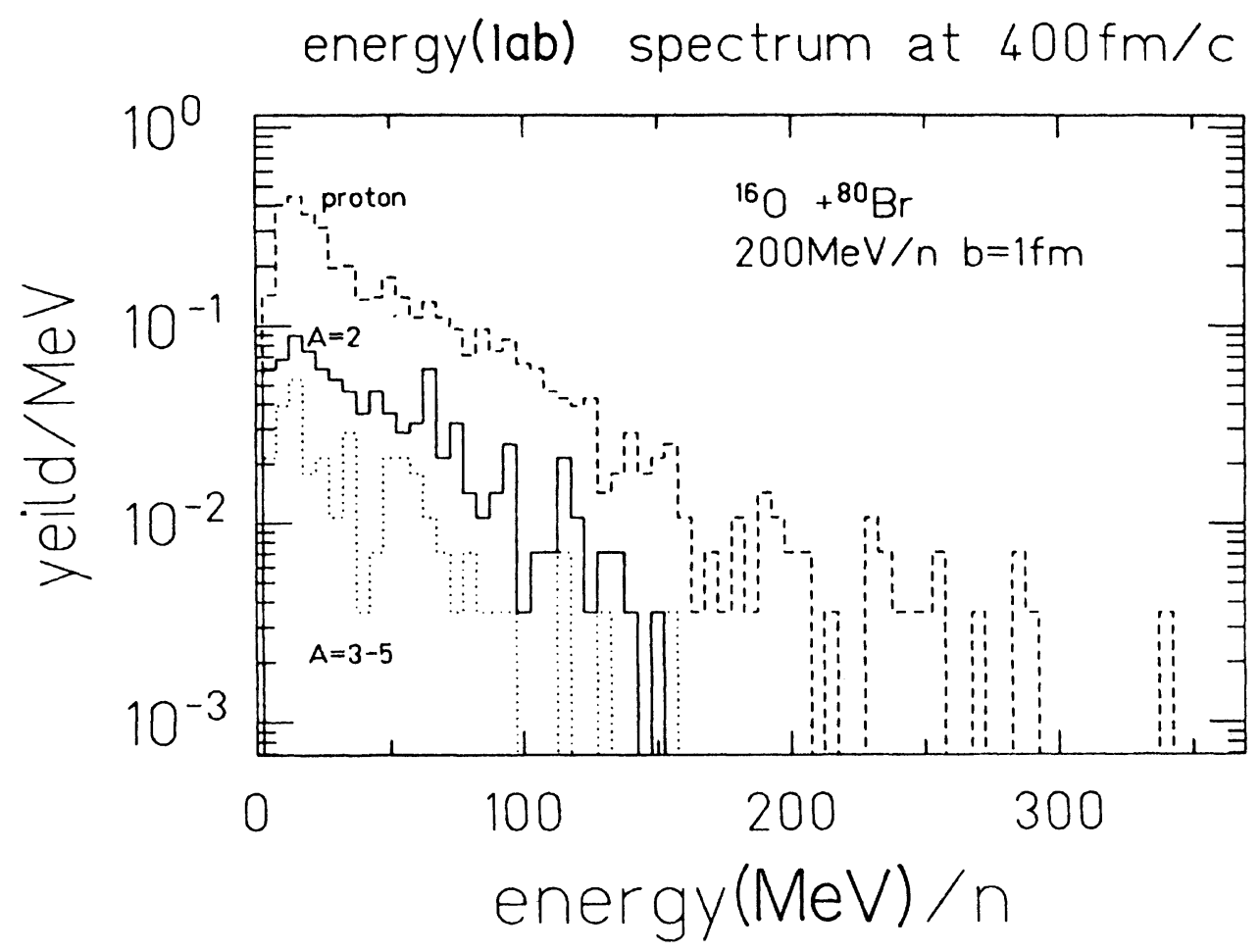

FIG. 10. Energy distribution of light complex fragments $(2 \leq A \leq 5)$ at incident energy $200 \mathrm{MeV} /$ nucleon.

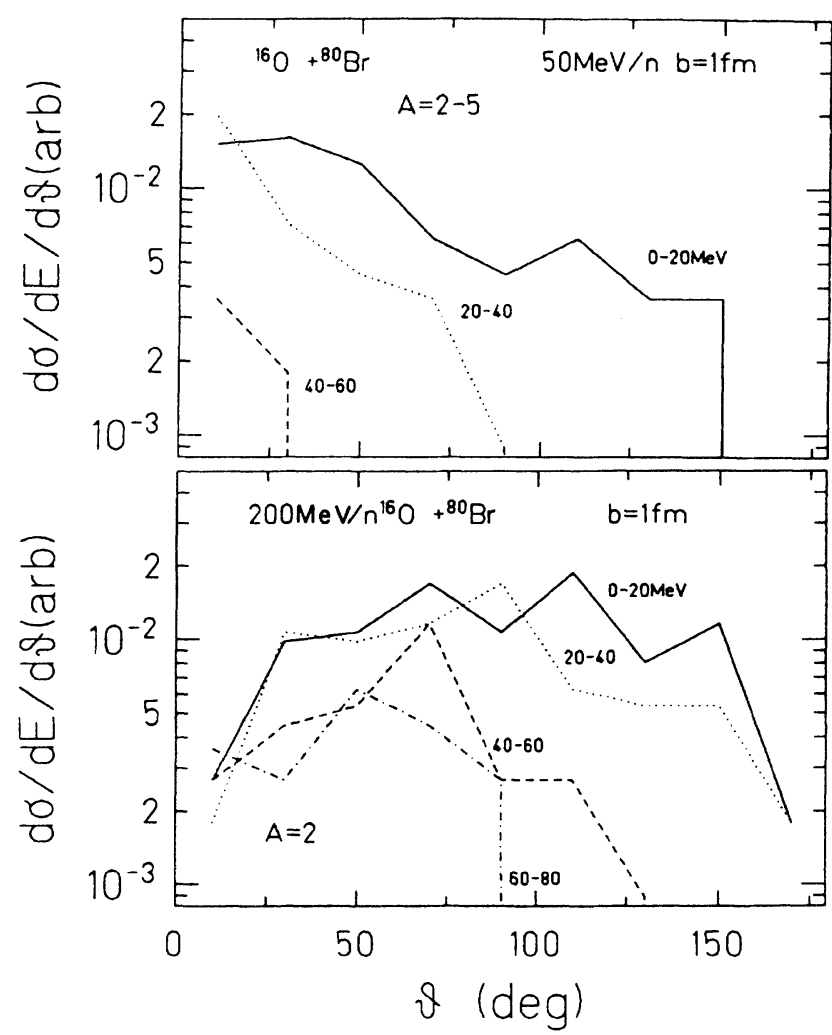

FIG. 11. Double-differential cross section of light complex fragments at 50 and $200 \mathrm{MeV} /$ nucleon.

\section{THE INFLUENCE OF PAULI POTENTIAL AND DAMPING PROCEDURE}

We extended the QMD model by a Pauli potential to ensure that the Pauli principle is fulfilled and introduced a damping procedure into the initialization procedure to get rid of the artificial excitation energy present in older implementations. It is therefore interesting to investigate the influence of these improvements. In Table II we compare the predictions of the QMD model for the four different possibilities of switching the Pauli potential and the damping procedure on and off. The damping procedure [3] consists of letting each nucleus at rest propagate for some time according to damped equations of motion, thus dissipating artificial excitation energy that stems from the random sampling initialization.

We compare the multiplicities of nucleons $M_{\text {nuc }}$, light complex fragments $M_{\mathrm{LCF}}(2 \leq A \leq 4)$, and intermediatemass fragments $M_{\mathrm{IMF}}(5 \leq A \leq 40)$ as calculated by the four different versions of the QMD model mentioned above.

Even if the numbers given in Table II are not very accurate because of bad statistics, their interpretation should be clear enough: The big discrepancy between the results for $M_{\text {nuc }}$ of the calculations with and without damped initialization could be expected. It is well known that the computational nuclei created by the random sampling procedure are not in the ground state, but are considerably excited (and even not stable for light nuclei). Therefore the damping procedure is very important for 
TABLE II. Multiplicity of nucleons, light complex fragments (LCF, $2 \leq A_{f} \leq 4$ ), and intermediate-mass fragments (IMF, $5 \leq A_{f} \leq 40$ ) for four different options, namely, with (On) and without (Off) Pauli potential and with (damping) and without (random sampling) initial cooling (damping) of the system. Left-hand side results for a calculation at $200 \mathrm{MeV}$, right-hand side a calculation for $25 \mathrm{MeV}$, both for central collisions ( $b=1 \mathrm{fm}$ ).

\begin{tabular}{|c|c|c|c|c|c|c|c|}
\hline \multirow{2}{*}{$\begin{array}{l}\text { Opt. } \\
V_{\text {Pauli }}\end{array}$} & \multirow{2}{*}{$\begin{array}{c}E_{\text {beam }}=200 \\
\text { Fragment } \\
\text { type }\end{array}$} & \multicolumn{2}{|c|}{$\begin{array}{r}\mathrm{MeV} / \text { nucleon, } b=1 \mathrm{fm} \\
\text { Multiplicity }\end{array}$} & \multirow{2}{*}{$\begin{array}{l}\text { Opt. } \\
V_{\text {Pauli }}\end{array}$} & \multirow{2}{*}{$\begin{array}{c}E_{\text {beam }}=25 \\
\text { Fragment } \\
\text { type }\end{array}$} & \multicolumn{2}{|c|}{$\begin{aligned} & \mathrm{MeV} / \text { nucleon, } b=1 \mathrm{fm} \\
& \text { Multiplicity }\end{aligned}$} \\
\hline & & Damping & Random sampling & & & Damping & Random sampling \\
\hline \multirow{3}{*}{ On } & Nucleons & 41.0 & 49.9 & \multirow{3}{*}{ On } & Nucleons & 11.00 & 18.60 \\
\hline & LCF & 6.9 & 6.3 & & LCF & 1.00 & 1.80 \\
\hline & IMF & 1.5 & 1.9 & & IMF & 0.87 & 0.20 \\
\hline \multirow{3}{*}{ Off } & Nucleons & 49.7 & 62.2 & \multirow{3}{*}{ Off } & Nucleons & 10.0 & 22.0 \\
\hline & LCF & 4.6 & 6.4 & & LCF & 1.2 & 1.8 \\
\hline & IMF & 1.5 & 1.5 & & IMF & 0.9 & 0.1 \\
\hline
\end{tabular}

low-energy heavy ion collisions, especially for light systems.

The Pauli potential introduced in our calculations separates the nucleons in phase space, thus leading to the fulfillment of the Pauli principle. This reduces the number of collisions. It seems, however, that this has no pronounced effect on the multiplicities. But it may have a more pronounced effect on quantities more sensitive to many-body correlations such as, e.g., angular distributions and energy spectra. This, however, has not been investigated yet and will be left to further study.

\section{CONCLUSIONS}

In the present work we have studied the transition from fusion-fission phenomena at $\approx 20 \mathrm{MeV} /$ nucleon to multifragmentation at 100-200 MeV/nucleon in collisions of ${ }^{16} \mathrm{O}$ on ${ }^{80} \mathrm{Br}$ employing the QMD model. The time evolution of density and mass distribution, chargedparticle multiplicity, and energy spectra as well as angular distributions of light particles have been investigated. All those quantities clearly exhibit that character of the reaction changes during the transition from lower to higher beam energies.

In the low-energy domain the reaction is dominated by fusion-fission phenomena. The fused system vibrates for a long time and then decays via binary channels. This can be deduced not only from the multiplicities, but also from the spectra and angular distributions of light particles.

For higher incident energies the mean density does not vibrate anymore, but drops dramatically after reaching its maximum. Correspondingly, the disassembly process gradually changes from binary decay to multifragmentation at higher energies. The multiplicity of intermediatemass fragments increases and spectra and angular distributions change shape. However, we do not find any sharp transition between the two decay modes mentioned above, but all quantities change gradually with energy. The calculated charged-particle multiplicities at various energies are in good agreement with recent experimental $4 \pi$ data.

We propose to measure spectra and angular distributions of light particles in the energy range considered here, because these might help to study the transition of the disassembly process in greater detail. Concerning the model itself, the influence of Pauli potential and damping procedure have been discussed. The introduction of the damped initialization is found to be crucial for lowenergy collisions, especially for light systems. The influence of the Pauli potential needs further study.

\section{ACKNOWLEDGMENTS}

The authors gratefully acknowledge valuable support from G. Peilert. L.Z. acknowledges the support of the Alexander von Humboldt Foundation. This work was supported by the Deutsche Forschungsgemeinschaft (DFG), by the Bundesministerium für Forschung and Technologie (BMFT), and by the Gesellschaft für Schwerionenforschung (GSI).
"Permanent address: Institute of Atomic Energy, P.O. Box 275, Beijing, China.

[1] R. Trockel, K. Hildenbrand, U. Lynen, W. F. J. Müller, H. J. Rabe, H. Sann, H. Stelzer, W. Trautman, and R. Wada, Phys. Rev. C 39, 729 (1989); B. Suraud, Ch. Gregoire, and B. Tamain (unpublished).

[2] B. Jakobsson, G. Jönsson, L. Karlsson, V. Kopljar, B. Noren, K. Söderström, F. Schussler, E. Monnand, H. Nifenecker, G. Fai, J. P. Bondorf, and K. Sneppen, Nucl. Phys. A509, 195 (1990).
[3] C. Hartnack, Li Zhuxia, L. Neise, G. Peilert, A. Rosenhauer, H. Sorge, J. Aichelin, H. Stöcker, and W. Greiner, Nucl. Phys. A495, 303(c) (1989); J. Aichelin, C. Hartnack, A. Bohnet, Li Zhuxia, G. Peilert, H. Stöcker, and W. Greiner, Phys. Lett. B 214, 34 (1989).

[4] C. Dorso, S. Duarte, and J. Randrup, Phys. Lett. B 188, 287 (1987); L. Wilets, E. M. Henley, M. Kreft, and A. D. Mackellar, Nucl. Phys. A282, 341 (1977).

[5] G. Peilert, J. Randrup, H. Stöcker, and W. Greiner, Phys. Lett. B (in press). 
[6] D. H. Boal and J. N. Glosli, Phys. Rev. C 37, 91 (1988); D. H. Boal and J. C. K. Wong, ibid. 41, 118 (1990).

[7] J. Konopka, G. Peilert, H. Stöcker, W. Greiner, and L. Neise, in Proceedings on the XXIXth Winter Meeting, Bormio (Italy), 1991, edited by I. Iori (Ricerca Scientifica ed Educazione Permanente, in press).

[8] G. Peilert, A. Rosenhauer, J. Aichelin, H. Stöcker, and W. Greiner, Phys. Rev. C 39, 1402 (1988); Mod. Phys. Lett. A 3, 459 (1988); Phys. Rev. Lett. 58, 1926 (1987).

[9] C. Ngô, S. Leray, M. E. Spina, H. Ngô, B. Remaud, and F.
Sebille (unpublished).

[10] B. Borderir, J. Phys. (Paris) Colloq. 47, C4-251 (1986), and references therein; B. Borderir, M. Montoya, M. F. Rivet, D. Jouan, C. Cabot, H. Fuchs, D. Gardes, H. Gauvin, D. Jacquet, F. Monnet, and F. Hanappe, Phys. Lett. B 205, 26 (1988).

[11] H. G. Baumgardt, J. U. Schott, Y. Sakamoto, E. Schopper, H. Stöcker, J. Hoffman, W. Scheid, and W. Greiner, Z. Phys. A 73, 359 (1975). 